\title{
REDES DE INNOVACIÓN DE LA ZARZAMORA EN MICHOACÁN EN EL CONTEXTO DE LOS MERCADOS INTERNACIONALES
}

\author{
INNOVATION NETWORKS OF THE BLACKBERRY IN MICHOACÁN IN \\ THE CONTEXT OF INTERNATIONAL MARKETS
}

\author{
${ }_{1}^{1}$ Zoe Infante Jiménez, ${ }^{2}$ Priscila Ortega Gómez, ${ }^{3}$ Federico Paredes Mejía
}

\begin{abstract}
RESUMEN
El propósito de la presente investigación es identificar y analizar la red de valor e innovación considerando la red social, de conocimiento y tecnológico dentro del plan de gestión de la innovación de zarzamora en el municipio de Los Reyes, Michoacán, distinguiendo dentro de esta el papel que desempeña la empresa Productores Agropecuarios por la Calidad (PROCAL) como una Sociedad de Producción Rural de Responsabilidad Limitada. Con esta finalidad, se aplicó la metodología de redes de innovación, que se basa en el proceso para la gestión de la innovación, así como en las etapas del ciclo del proyecto. En base a los resultados obtenidos, se pudo concluir que existe gran diversidad de productores con distintas capacidades tecnológicas asociadas a diversos patrones de producción y distribución del conocimiento tácito; algunos con altas habilidades para socializar el conocimiento de difusión, otros con altas capacidades para producir, pero sin socializar el conocimiento tácito. Dentro de la red de valor, el papel de PROCAL se centra en funcionar como complementador, no obstante, su actuación debe ser reforzada con bases sólidas para contribuir al favorecimiento de los diversos eslabones que componen la red; lo que se verá reflejado en un mayor beneficio para la empresa. Para ello, debe continuar reforzando sus vínculos con las diferentes instituciones gubernamentales y privadas, así como con los centros de investigación y de fomento.
\end{abstract}

Palabras clave: Red de valor, redes de innovación, ventajas competitivas, zarzamora.

\begin{abstract}
The purpose of this research is identify and analyze the value and innovation network considering the social, knowledge and technological network within the innovation management plan of zarzamora in the municipality of Los Reyes, Michoacán, distinguishing within this the paper carried out by the company Agricultural Producers for Quality (PROCAL), which is a Rural Production Company with Limited Responsibility. The methodology of innovation networks was applied, which is based on the process for the management of innovation, as well as in the stages of the project cycle. Based on the results obtained, it can be concluded that there is a great diversity of producers with different technological capabilities associated with different patterns of production and distribution of this tacit knowledge; some with high skills to socialize knowledge of diffusion, others with high capacity to produce, but without socializing tacit knowledge. Within the value network, the role of PROCAL is focused on functioning as a complement, however, its action must be reinforced with solid foundations to contribute to the promotion of the various links that make up the network, which in turn, will be reflected in a greater benefit for the company, for this, it must continue to strengthen its links with the different governmental and private institutions, as well as research and development centers.
\end{abstract}

Keywords: Value network, networks of innovation, competitive advantages, blackberries.

\footnotetext{
IDocente investigador. Universidad Michoacana de San Nicolás de Hidalgo. Michoacán - México. E-mail: zoeinfantejimenez@yahoo.com.mx 2Docente investigador. Universidad Michoacana de San Nicolás de Hidalgo. Michoacán - México. E-mail: prisog@hotmail.com 3Docente investigador. Universidad Michoacana de San Nicolás de Hidalgo. Michoacán - México. E-mail: fparedes@fira.gob.mx
} 


\section{INTRODUCCIÓN}

La innovación es una condición fundamental para el crecimiento económico sostenido; es una herramienta indispensable para lograr sistemas de producción sustentables en el medio rural mexicano, reduce las disparidades sociales y permite un uso sostenible de sus recursos naturales (Aguilar, 2004; Aguilar et al., 2010). Las redes de valor toman en cuenta los nuevos criterios de competencia, proporcionando una nueva forma de competir que considere la transferencia de tecnología y la asistencia técnica. Coángelo (2002) señala que la red de valor obtiene ventajas por medio de la circulación de conocimiento viable entre sus integrantes a través de los mecanismos de la integración. Para Cotec (2006), la meta de cualquier proceso innovador es la generación de valor. La difusión es el proceso mediante el cual las innovaciones se extienden a los diferentes consumidores, países, regiones, sectores, mercados y empresas, después de su primera introducción (OECD, 2005), por lo tanto, la red de comunicación determinará la rapidez con la cual se pueden difundir las innovaciones y ser adoptadas por cada individuo.

Actualmente, el crecimiento de las empresas se basa en lograr obtener ventaja de la innovación tecnológica. También es necesario una organización adecuada para aprovecharla (Pérez, 2009). Respecto a la demanda, la ventaja competitiva debe estar claramente diferenciada frente a los competidores. La "innovación en valor" (Kim y Mauborgue, 2006).

El estado de Michoacán es el principal productor y exportador de zarzamora representando el $98 \%$ de la producción nacional; el cultivo de la zarzamora ha tenido un desarrollo acelerado, con una tasa media anual de crecimiento del $69 \%$. En la actualidad, Michoacán tiene una superficie estimada de zarzamora de 6,482 hectáreas (ha), siendo el municipio de Los Reyes, el que concentra la mayoría de la superficie cultivada con $5 \mathrm{mil} 250 \mathrm{ha}$, donde laboran un promedio de mil 833 productores y genera alrededor de 5,000 empleos permanentes y 10,000 temporales, considerándose así, la tercera derrama económica agrícola.

El desarrollo acelerado de este cultivo se debe al precio alto del producto de exportación y a la aceptación del producto en el principal mercado de exportación (Estados Unidos), por las ventajas comparativas en la cercanía geográfica y a la cosecha en épocas donde los mercados internacionales no ofertan el producto. Sin embargo, para ser exportada, la zarzamora debe cumplir con un alto grado de calidad para cumplir con las BPA (buenas prácticas agrícolas internacionales), existiendo el rechazo del producto en un promedio del $10 \%$.

En Los Reyes se encuentra la empresa conformada por productores, denominada Sociedad de Producción Rural de Responsabilidad Limitada (PROCAL), la cual oferta diferentes servicios y productos, desempeñando varios roles dentro de la red de Valor de zarzamora. Uno de estos roles es como complementador, ofreciendo el servicio de asistencia técnica a los productores con predios de tamaño mediano y chico, para el mejoramiento en la calidad de la zarzamora a través de innovaciones en la producción.

Específicamente se pretende conocer la estructura relacional de los actores y sus vinculaciones en la Red de zarzamora, a) Identificando el comportamiento de la red y de los nodos en lo individual; b) Valorando el desempeño de los actores y obteniendo una perspectiva de grupo, analizando a los demás actores de la red; c) Conociendo las características de la red: su caracterización, su densidad y su centralidad. En base a todo esto, se diseña una estrategia de intervención que contribuya a que los productores incrementen la calidad.

\section{MATERIALES YMÉTODOS}

El presente estudio se llevó a cabo en una región conformada por seis municipios donde tiene presencia la empresa PROCAL: Los Reyes, Tocumbo, Periban, Tancítaro, Cotija y Uruapan.

Se aplicó la metodología de redes de innovación, basada en el proceso para la gestión de la innovación y las etapas del ciclo del proyecto que realizan las Agencias para la Gestión de la Innovación (AGI). En primer lugar, se llevaron a cabo visitas y entrevistas a los dirigentes de la empresa PROCAL, SPR., a los principales centros de acopio (Sunbell, El Cerrito, S.P.R.L, y a Driscoll), al representante del área coordinadora de la Universidad Michoacana de San Nicolás de Hidalgo, al personal de Banco de MéxicoFIRA de la Dirección Regional Occidente y a la Agencia Zamora. En seguida, se aplicó el método de marco lógico mediante talleres para el análisis de los factores críticos en los que participaron directivos de la empresa PROCAL, SPR., productores socios y clientes de esta empresa; con ello se obtuvo el análisis de involucrados, el árbol de problemas, el árbol de soluciones, el análisis de alternativas, la matriz del marco lógico, el modelo de inversión para el mejoramiento técnico y a partir de todos ellos se determinaron lineamientos de la estrategia de intervención.

Posteriormente, se realizó la encuesta de la línea base incorporando datos de una muestra de 19 productores de un universo de 90 productores de zarzamora, una parte de estos son socios y otros clientes de la empresa PROCAL, los cuales tienen sus predios en diferentes municipios del estado de Michoacán. La encuesta de línea base contempla aspectos generales, red social, aspectos técnicos, perfil y dinámica de la actividad, superficie, percepción y aspectos económicos, valoración de problemas, financiero y económico, 
recursos económicos, percepción de trámite ante los intermediarios financieros (IF's), inversiones conjuntas, equipos, instalaciones e infraestructura, requerimientos de insumos, infraestructura y equipo comercial, venta de producción, compra de insumos, dinámica de innovación, organización. Finalmente, se realizaron los cálculos de la línea base de INAE, TAI, brechas de innovación y el mapeado de las siguientes redes sociales: aprendizaje, comunicación, financiera, proveedores y organización.

\section{RESULTADOS Y DISCUSIÓN}

Con los resultados obtenidos mediante el método de marco lógico, como parte de los lineamientos de la estrategia de intervención para la red de valor, se determinaron 3 alternativas: de tipo tecnológico, para el desarrollo de tecnologías de alta productividad, y para mejorar la rentabilidad.

Algunos atributos y dinámica de la población son: la población analizada tiene una edad promedio de 46,4 años, mayoritariamente hombres $(89 \%)$, un grado de escolaridad promedio de 12,6 años, la experiencia media en la actividad es de 8,4 años. Respecto a la dinámica de las actividades, la tenencia de la tierra es $100 \%$ propiedad privada; superficie total dedicada a la producción de zarzamora: 11,9 ha y la superficie cultivada: 6,5 ha. Edad promedio de la planta y productividad: 6,1 años y 3,868.4 cajas por ha, (casi 9 toneladas/ha) en ambos casos con un coeficiente de variación muy bajo.

La variedad de zarzamora mayormente cultivada es la Tupi. El 47\% de los encuestados genera entre el 51 al $100 \%$ de los ingresos y el $26 \%$ están de tiempo completo en el cultivo de la zarzamora. Los productores restantes dedican tiempo parcial al cultivo, en tanto desarrollan otra actividad agrícola como el cultivo de aguacate y/o durazno, por lo que sus ingresos por concepto de este cultivo varían desde el 25 al $75 \%$.

En cuanto a la comercialización y ventas, el $92 \%$ de la producción es destinada a la exportación; el resto no cumple las especificaciones de calidad, comercializándose en el mercado nacional. Las empresas extranjeras Sunbelle, Splendor y Driscoll acopian la producción en un $80 \%$ de la población de análisis. Las empresas nacionales El Molinito y Moradely acopian el $75 \%$ del producto que se comercializa en el mercado nacional. En cuanto a la percepción del negocio y de la problemática del entorno que muestran los productores, $63 \%$ la percibe con optimismo; dentro de este grupo el $58 \%$ la ve en crecimiento, $32 \%$ la percibe como estancada y el $6 \%$ cree que está decreciendo. Por lo que consideramos este rubro como un ambiente optimista. Los problemas que afrontan en la producción con alto riesgo de incidencia son: costos de los insumos, costo de la mano de obra, bajos precios, calidad de los caminos, así como plagas y enfermedades; los problemas con una incidencia menor radican en la variedad de la planta. El servicio de asistencia técnica, la disponibilidad de agua y los bajos rendimientos son catalogados como problemas de riesgo medio. En cuanto a las necesidades de inversión y tipo de crédito existe un requerimiento de capital de trabajo de 6,8 millones $(38,7 \%)$ y el resto, $61,3 \%$ de crédito refaccionario. La fuente más importante de financiamiento son las empresas acopiadoras-comercializadoras, ya que actúan como parafinancieras otorgando el capital de trabajo para asegurar la proveeduría demandada para la comercialización. Debido a que la red se encuentra muy desarrollada en la región, el productor tiene a su disponibilidad todas las empresas para proveerse de los insumos requeridos, pero solo el $31 \%$ de los productores se encuentra organizado para comprar los insumos de forma consolidada. El cultivo es altamente rentable y ha representado una excelente alternativa para productores minifundistas. El cultivo es perenne y el mayor costo de inversión se observa en el establecimiento del cultivo asegurando el sano desarrollo del mismo; la recuperación de la inversión inicial se logra en la mayoría de los casos desde el primer ciclo o al segundo año de producción.

\section{Análisis de la dinámica de innovación}

La relación de innovaciones está conformada por nueve grandes categorías, que a su vez contienen 28 variables específicas: 1. Nutrición: Análisis foliar, aplicación de fertilizantes, fertilización foliar, fertilizantes orgánicos; 2. Sanidad: monitorio de plagas, podas sanitarias, calibración de equipos control de agua; 3. Manejo sostenible de recursos: Recolecta envases de químicos, control biológico y enfermedades, uso de macro túneles; 4 . Establecimiento y manejo de la plantación: uso de estimulantes y sistemas de riego; 5. Cosecha: Cortes por criterio madurez, cortes criterio calidad, acondicionamiento del producto, programa de cosecha, trazabilidad, instalaciones sanitarias; 6 . Reproducción y genética: otras especie y uso de variedades mejoradas; 7. Administración: Calendario de actividades, registro de prácticas efectuadas, registro de ingresos y egresos, contrato de asistencia técnica; 8. Organización: Esquema de articulación con agroindustriales; y 9. Gestión: Gestión conjunta.

Los productores de zarzamora entrevistados para establecer la Encuesta de Línea Base (ELB) están ubicados en el valle de Los Reyes en Michoacán, que es el área de influencia de tres municipios: Los Reyes, Peribán y Tocumbo.

En cuanto al Índice de Adopción de Innovaciones (INAE) de los productores, aproximadamente el $50 \%$ de los entrevistados se encuentran arriba del promedio general $(79 \%)$. Por otra parte, se tiene identificado al productor con un alto porcentaje en la innovación tecnológica. Tres categorías presentan el mayor rezago y por tanto demandan una atención adecuada. Estas categorías sirven de base para establecer la Estrategia de Innovación que permita elevar el INAE. 
En materia de organización los productores perciben la necesidad de organizar la compra de insumos y acceder a insumos inocuos para generar economías de escala. La segunda categoría más sentida como problemática por parte de los productores es la reproducción de plántula y el manejo genético de la misma.
Actualmente todos los productores tienen establecida la variedad Tupi. Solamente uno de ellos tiene de manera adicional una nueva variedad: la sliping bioner. Esta innovación no repercute en el cumplimiento de las especificaciones de los comercializadores.

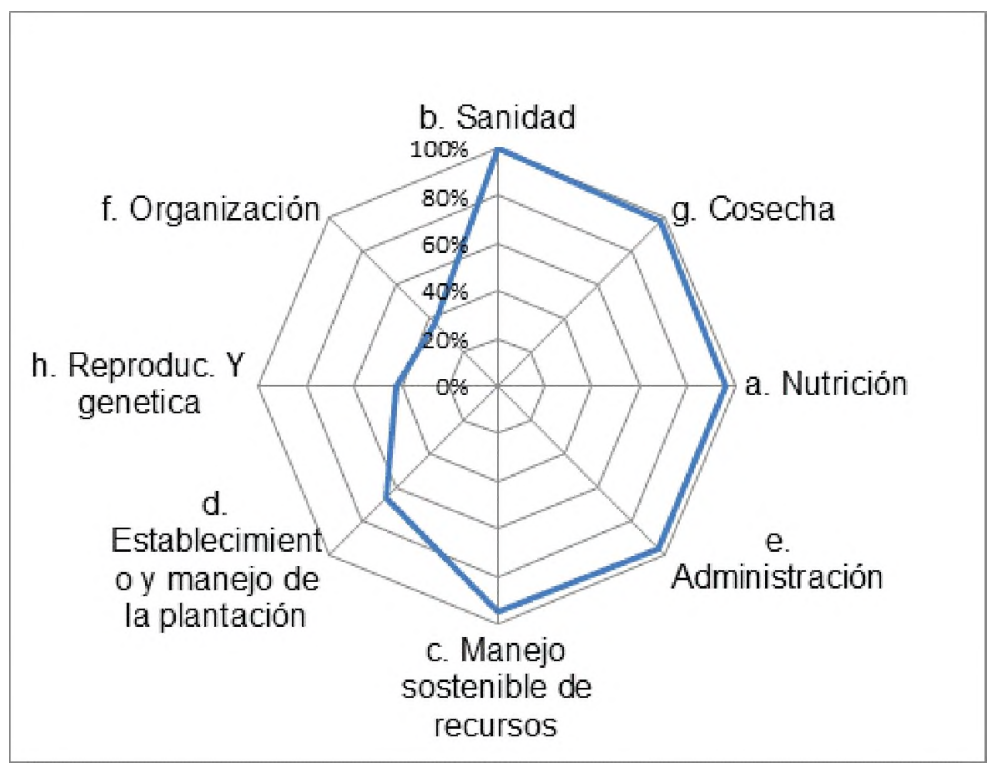

Figura 1. Perfil del Índice de Adopción de Innovaciones de los productores.

Fuente: Elaboración propia con datos de la encuesta ELB.

En cuanto al establecimiento y manejo de la plantación, el productor de zarzamora del valle de Los Reyes identifica que establecer un cultivo sano y vigoroso, además de manejar adecuadamente la huerta, son condiciones indispensables para mantenerse en el mercado. Las innovaciones con menor tasa de adopción son: manejo del sistema de riego y esquema de articulación agroindustrial, uso de variedades mejoradas, contratación de asistencia técnica, aplicación de fertilizantes específicos y el uso de macrotúneles.

A manera comparativa se analizaron las innovaciones con altas y menores tasas de adopción, con el objetivo de analizar el nivel de madurez que han adquirido las innovaciones y mostrar el área de oportunidad para incrementar el uso de cada una de ellas en caso de que las evidencias indiquen la factibilidad de su difusión (figura 2).

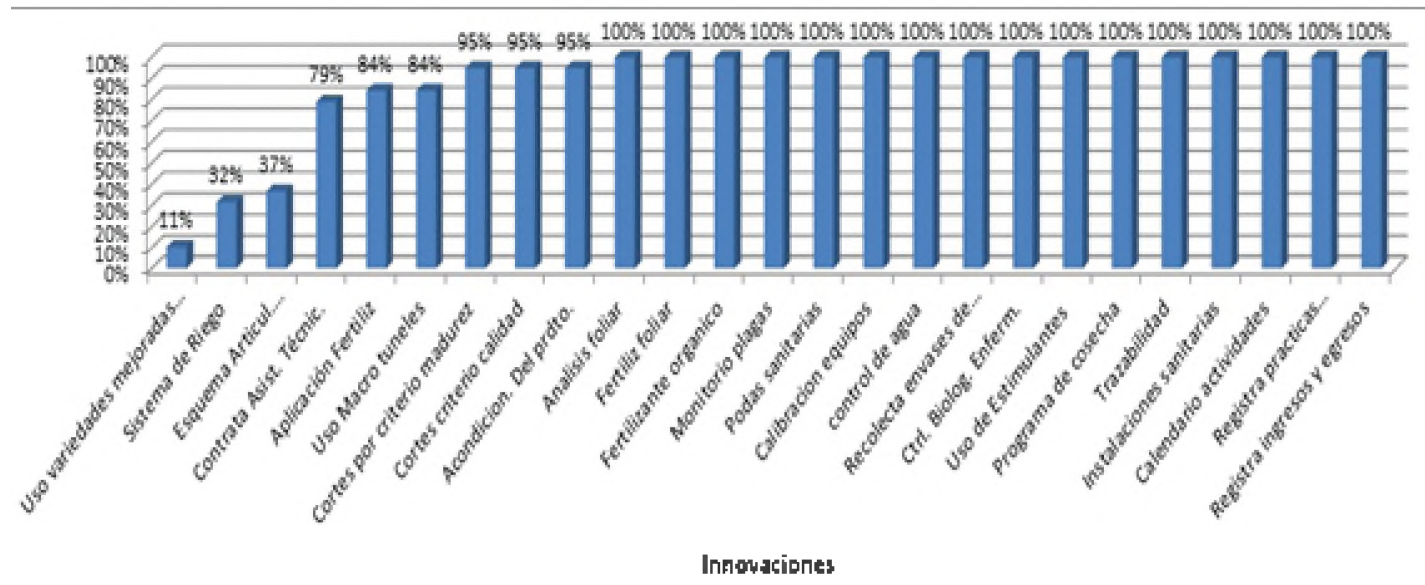

Figura 2. Tasa de Adopción de Innovaciones (TAI) de los productores.

Fuente: Elaboración propia con datos de la encuesta ELB. 
En cuanto a la innovación en nutrición con valor alto en el TAI, su adopción del 100\% fue en doce años; sin embargo, la innovación empezó a tener aceptación en una masa crítica de adoptantes en un periodo de cinco a seis años. Respecto a la innovación en la categoría de sanidad, que también tiene un valor alto de TAI, el periodo de adopción al $100 \%$ transcurrió en once años; no obstante, la masa crítica adoptante fue en un periodo de 5-6 años. En ambos casos, la adopción fue influenciada y catalizada por el interés del productor al tener la necesidad de una mayor producción y tendencia hacia actores determinados. Estos valores se explican así por el número de productores encuestados y tipo de respuesta proporcionada. El mapeo reveló los tipos de productores y los roles en cada red, por su parte, el uso de sistemas de riego que tiene un valor bajo de TAI ha tenido una lenta adopción, en el transcurso de once años aún no ha alcanzado la masa crítica de adoptantes.

Actualmente se están presentando problemas con la disponibilidad de agua, tanto en cantidad como en calidad, debido a la demanda de este líquido por parte de los servicios urbanos y para el uso agrícola, este hecho será uno de los factores que catalizará la adopción de esta innovación.

\section{Mapeo de la red y el análisis gráfico de la red de innovación}

El mapeo se realizó a cinco tipos de redes: sociales, financiera, de comercialización, proveeduría y la de organización; se obtuvo un total de diecisiete aspectos considerados. Adicionalmente se mapea la combinación de tres redes, con lo cual se estableció la posición de los actores más influyentes.

Tabla 1. Resultados de los principales indicadores de las redes mapeadas.

\begin{tabular}{|c|c|c|c|c|c|c|c|}
\hline \multirow{2}{*}{ Tipos de redes } & & \multirow{2}{*}{ Densidad } & \multicolumn{2}{|c|}{ Centralidad } & \multicolumn{3}{|c|}{ Centralización(intermediación) } \\
\hline & & & Salida & Entrada & Índice & Actor Ppall & Valor \\
\hline \multirow{3}{*}{ Social } & Con quién habla & $4,50 \%$ & 9,19 & 1,5 & 0,67 & Ere03 & 5 \\
\hline & De quién aprende & $3,23 \%$ & 1,66 & 8,32 & 0 & ERELT04 & 0 \\
\hline & Innovadores & $7,14 \%$ & 1,55 & 33 & 2,08 & Ere03 & 8 \\
\hline Financiamiento & Avío & $6,32 \%$ & 1,66 & 8,79 & 0 & PF02 & 0 \\
\hline \multirow{2}{*}{ Comercialización } & Exportación & $5,23 \%$ & 1,44 & 34,72 & 0 & PF02 & 0 \\
\hline & Mercado nacional & $3,62 \%$ & 2,64 & 20,79 & $\mathbf{0}$ & CA02 & $\mathbf{0}$ \\
\hline \multirow{3}{*}{ Proveeduría } & $\begin{array}{l}\text { Compra insumos } \\
\text { principal }\end{array}$ & $7,62 \%$ & 1,22 & 38 & $\mathbf{0}$ & P101 & $\mathbf{0}$ \\
\hline & $\begin{array}{l}\text { Compra insumos } \\
\text { secundario }\end{array}$ & $4,92 \%$ & 1,66 & 9,92 & $\mathbf{0}$ & - & $\mathbf{0}$ \\
\hline & Gestión conjunta & $8,19 \%$ & 1,23 & 30,55 & 0,65 & OP1 & 2 \\
\hline Organización & $\begin{array}{l}\text { Pertenece a una } \\
\text { organización }\end{array}$ & $8,82 \%$ & 1,95 & 48,43 & $\mathbf{0}$ & OP1 & $\mathbf{0}$ \\
\hline \multirow{3}{*}{ Combinación } & Habla + aprende & $3,66 \%$ & 1,53 & 2,83 & 0,62 & Varios & 10 \\
\hline & $\begin{array}{l}\text { Habla + aprende + } \\
\text { innovación }\end{array}$ & $3,94 \%$ & 2,24 & 10,38 & 1,4 & Varios & 27 \\
\hline & $\begin{array}{l}\text { Habla }+ \text { aprende }+ \\
\text { innovación }+ \\
\text { gestión }\end{array}$ & $4,53 \%$ & 1,94 & 7,74 & 1 & Varios & 22 \\
\hline
\end{tabular}

Fuente: Elaboración propia con datos de la encuesta ELB.

En la red de aprendizaje se presenta una gráfica que se aproxima al tipo PC, siendo una red con un ámbito de estructuración de tipo difusa. La densidad es muy baja, identificándose un grupo pequeño con un actor ERELT04 (colector), con grado de entrada tres (prestigio) y estructurador que es un productor identificado como líder tecnológico.

La red de comunicación es caracterizada por una gráfica que se aproxima al tipo PC y con un ámbito de estructuración de tipo difusa. Sus relaciones (densidad) son bajas, pero se determina claramente una agrupación donde se identifica al productor Ere03 como un actor fuente, buscador de información (grado de salida tres) y difusor. Además, presenta un valor de cinco en el indicador de intermediación. Este es un productor referido y dirigente de la empresa PROCAL.

En la red de financiamiento se presenta una gráfica que se aproxima al tipo PC y es una red con un ámbito de estructuración de tipo difusa. Destacan tres pequeños grupos, teniendo actores como colectores (con grado de entrada de dos y tres), de prestigio y sin ser difusores o estructuradores. De estos dos actores, donde la empresa comercializadora Sunbelle (PF02) actúa como intermediario financiero y al mismo tiempo 
como acopiador, el otro actor es un intermediario financiero no bancario: Caja Popular (PF03). Esta red no tiene valor para el indicador de Intermediación.

En las redes de comercialización, tanto para mercado de exportación como para el mercado nacional, se forma una gráfica que se aproxima al tipo PC con un ámbito de estructuración de tipo difusa. Destacan dos actores colectores: para el mercado nacional la empresa El Molinito (CA02, con grado de entrada cinco) y para la exportación Sunbelle (PF02), que domina la preferencia de los productores (con grado de entrada nueve); en ambos casos son actores de prestigio y sin ser difusores o estructuradores. Esta red no tiene valor para el indicador de Intermediación.

En la red de proveeduría de insumos, se presenta la participación con un grado de entrada significativa (ocho) del actor para los insumos principales (PI01); se trata de la empresa de fertilizantes Agro fertilizantes de Michoacán, un actor de tipo colector, de prestigio y sin ser difusor o estructurador.

En la red de innovación aparece con un número importante de entradas (siete), el productor referido Ere03 que funge como un actor colector y difusor, tiene un valor importante (ocho) como intermediación es referido por un número importante de productores como el innovador.
Para las redes de gestión de insumos y servicios - así como de organización-, el actor principal es OP1 (PROCAL) que funge como un actor colector (grado de entrada seis) y difusor. En el caso de la red de insumos tiene un valor de dos como intermediación.

Mediante el mapeo conjunto en la red de comunicación, de aprendizaje, innovación y de gestión se obtienen tres mapeos: El primero, de la red de comunicación con la de aprendizaje; el segundo, de la red de comunicación con la de aprendizaje y con la de innovación; el tercero, de la red de comunicación, de aprendizaje, de innovación y de gestión.

En estos tres mapas, las relaciones entre los diferentes actores se hacen más evidentes y claras. Los indicadores de centralización, entradas y salidas —así como de intermediación - toman valores más altos, definiendo el papel de productores y empresas claves (Ere03 y OP1) como actores fuentes, colectores y articuladores. Además se observa que la red en los aspectos comunicación, aprendizaje, innovación y gestión tienen varias comunidades.

Del análisis de los indicadores y tomando en cuenta los resultados, tanto de centralización (en este caso, intermediación) como de centralidad para esta red combinada, se definen los actores para la estrategia de intervención.

Tabla 2. Indicadores de los actores principales de la red combinada.

\begin{tabular}{c|l|c|c|c}
\hline \multirow{2}{*}{ Actor } & \multicolumn{1}{|c|}{ Tipo de Actor } & \multicolumn{2}{c|}{ Centralidad } & \multirow{2}{*}{ Intermediación } \\
\cline { 3 - 4 } & & Salida & Entrada & \\
\hline Ere03 & Productor referido & 4 & 12 & 22 \\
\hline ERe04 & Productor referido & 4 & 12 & 7 \\
\hline ER06 & Productor típico & 0 & 0 & 7 \\
\hline ER07 & Productor típico & 0 & 0 & 4 \\
\hline OP1 & Organización de productores & 0 & 7 & 0 \\
\hline ERELT04 & Productor líder tecnológico & 0 & 3 & 0 \\
\hline
\end{tabular}

Fuente: Elaboración propia con datos de la encuesta ELB.

Como primer término se identificaron a los dirigentes de la empresa PROCAL y a los productores referidos (Ere03 y Ere04), seguidos de dos productores típicos y de un productor líder tecnológico. Estos actores, tienen asimismo atributos de difusores, articuladores, colectores y buscadores de información.

\section{CONCLUSIONES}

La red productiva de zarzamora en la región de Los Reyes, Michoacán, presenta un considerable avance en su organización y su interacción respecto a otras redes en el país, ya que está debidamente estructurada con todos los actores en el área: los productores en forma individual como en forma organizada, los comercializadores, los industriales, los proveedores de insumos y de servicios en general. Sin embargo, mediante la aplicación de la ELB se identificaron diferentes características respecto a las innovaciones que requieren adoptar los productores para mejorar su productividad, así como para determinar las dinámicas de innovación y establecer lo requerido. Se plasmaron la dinámica de las actividades productivas, económicas y comerciales, junto con su percepción del negocio y de la problemática tanto de su entorno, como financiero. También se identificaron sus necesidades crediticias, la relación comercial con sus proveedores y los aspectos de su ambiente social. Con el análisis 
gráfico de la red se determinaron las diferentes características y actores más influyentes dentro de la comunidad, considerando estos últimos con un buen nivel tecnológico y con una buena disposición a comunicar sus conocimientos.

Dentro de la red de valor, la empresa PROCAL funge como complementador: brinda a socios y clientes diversos servicios y productos como abastecedor de insumos, unidad de capacitación y asistencia técnica, centro de investigación, unidad de producción, dispensadora de insumos, entre otros; no obstante, debe tener un desempeño más activo y contundente para desempeñar el papel de aglutinador en la organización de productores y mejorar la densidad de la red. La eficacia de estas acciones depende de seleccionar y consolidar los equipos técnicos, de asegurar que se apliquen metodologías de trabajo eficaces y orientadas a la rendición de cuentas y de involucrar a los actores locales para dar orientación estratégica y seguimiento a los procesos. PROCAL debe continuar reforzando sus vínculos con las diferentes instituciones gubernamentales y privadas, así como con los centros de investigación y de fomento.

\section{REFERENCIAS BIBLIOGRÁFICAS}

Aguilar, Á. J. (2011). Consultoría para apoyar el rediseño del subsistema nacional de asistencia técnica integral del ministerio de agricultura y desarrollo rural; Del extensionismo lineal a las redes de innovación agencias de gestión de la innovación. Universidad de Chapingo. México.

Aguilar, Á., J. (2011). Definiciones y conceptos básicos en la gestión de redes de innovación. Universidad Autónoma de Chapingo-CIESTAAM. México.

Aguilar, Á., J., J. R. Altamirano C., R. Rendón M. y V. H. Santoyo C. $(2010)$. De 1 extensionismo agrícola a las redes de innovación rural. CIESTAAM. México.

Aguilar, Á., J., M. Muñoz R., R. Rendón M. y J. R. Altamirano C. (2007). Selección de actores a entrevistar para analizar la dinámica de innovaciones bajo el enfoque de redes. Universidad Autónoma de Chapingo-CIESTAAM. México.

Ambriz, Z., F. J. (2009). Sistema Producto Zarzamora en Michoacán. Plan Rector 2010-2012. SAGARPA, Secretaría de Desarrollo Rural, Michoacán y SP Zarzamora Michoacán. Michoacán, México.

Briseño, G., J. A. y G. Calleros C. (2009). "Plan de negocios y diseño del diagnóstico de Productores Agropecuarios por la Calidad, SPR de RL". México.
Coángelo, L. (2002). "A evolução das redes de vaor integradas: um desafio a capacidade gerencial". Expo Management. Brasil.

CONAPO. (2002). Indicadores demográficos 2005 2030. CONAPO. México.

COTEC. (2007). La persona protagonista de la innovación. Fundación COTEC para la innovación tecnológica. Madrid, España.

Delgado, M., F. J., R. Vela G., H. Quintero L. (1992). "El riesgo en proyectos de inversión y alternativas para su disminución". FIRA: Boletín Informativo. Núm. 243, vol. XXV. México.

FAO. (2009). Cómo alimentar al mundo en 2050: la agricultura mundial en la perspectiva del año 2050.Secretaría del Foro de Alto Nivel de Expertos. FAO. Roma.

FIRA. (2008). Programa de Inducción y Desarrollo del Financiamiento al Medio Rural: Componente de Apoyo a instrumentos de inducción y Desarrollo del financiamiento. FINCAS. México.

Gobierno del estado de Chihuahua. (2002). Potencial agroindustrial para el cultivo de frutillas en el estado de Chihuahua. Gobierno del estado de Chihuahua. México.

Kim, W. C. y R. Mauborgne. (2005). La estrategia del océano azul: cómo desarrollar un nuevo mercado donde la competencia no tiene ninguna importancia. Grupo Editorial Norma. Bogotá.

Muñoz, R., M.; J. Aguilar Á., R. Rendón M. y J. R. Altamirano C. (2007). Análisis de la dinámica de innovación en cadenas agroalimentarias. Universidad Autónoma de Chapingo, CIESTAAM. México.

Muñoz, R., M., R. Rendón M., J. Aguilar Á., S. de la Cruz y J. R. Altamirano C. (2008). "Diseño del modelo de agencias para la gestión de la innovación en cadenas agroalimentarias". Informe final y productos del TCP/MEX/3103/MEX. México.

OCDE y EUROSTAT. (2005). Manual Oslo: guía para la recogida e interpretación de datos sobre innovación. 3ra Ed. Grupo TRAGSA.

Olamendi, G. (2012). "Estrategias de diferenciación". http://www.estoesmarketing.co m. Consultada 30 de octubre de 2012.

Osterwalder, A. y Y. Pigneur. (2010). Business model generation. Ed. PAPF, S.L.U. New Jersey, EE.UU.

Paredes, M., F., J J. Castellanos F. y F. Sánchez H. (2011). "Reporte de resultados del mercado de asesoría y consultoría en FIRA". Banco de México-FIRA. México. 
Pérez, C. (2009). “La otra globalización: los retos del colapso financiero", Problemas del Desarrollo. Revista Latinoamericana de Economia. 40: 11-37.

Rendón, M., R., J. Aguilar Á., J. R. Altamirano C. y M. Muñoz R. (2010). "El modelo de agencias para la gestión de la innovación". Universidad Autónoma de Chapingo-CIESTAAM. México.

Rendón, M., R., J. Aguilar Á., J. R. Altamirano C. y M. Muñoz R. (2009). Etapas del mapeo de redes territoriales de innovación. Universidad Autónoma ChapingoCIESTAAM. México.

Rendón, M., R., J. Aguilar Á., M. Muñoz R. y J. R. Altamirano C. (2007). Identificación de actores clave para la gestión de la innovación: el uso de redes sociales. Universidad Autónoma ChapingoCIESTAAM. México.

Rendón, M., R., M. Muñoz R., J. Aguilar Á. y J. R. Altamira C. (2007). Planeación de proyectos para gestionar la innovación. Universidad Autónoma Chapingo-CIESTAAM, México.

Ricart, C, J. E. (2009). "Modelo de negocios: el eslabón perdido en la dirección estratégica". Universia Business Review. 23:12-25.

Ricart, C, J. E. (2009). "Modelos de negocio para una nueva organización industrial". Colección de Papeles de Economía Industrial 28. Universidad de Empresa II. Cataluña, España.

Rogers, E. M. (1960). Social change in rural society: a textbook in rural sociology. New York, EE.UU.

Rogers, E. M. (2003). Diffusion of innovations. 5th ed. Free Press. New York, EE.UU.

SAGARPA. (2009). Plan Rector 2010-2012. Sistema Producto Zarzamora en Michoacán, A.C. SAGARPA. México.

Sánchez, G., J. (2012). "La adopción de innovaciones en agroempresas ovinas". Tesis de maestría. Universidad Autónoma de Chapingo, CIESTAAM. México.

Sánchez R., G. (2008). La red de valor de la zarzamora: el clúster de Los Reyes Michoacán, un ejemplo de reconversión competitiva. Sistema de Inteligencia de Mercados. Fundación PRODUCE Michoacán. México.

Santoyo, C., V. H. (2010). El rol de la agencia en el sistema de extensionismo e innovación y Subdirección de Desarrollo de Mercados de Asesoría y Consultoría. Universidad Autónoma de Chapingo, CIESTAAM. México.
Santoyo, C., V. H., M. Muñoz R., R. Rendón M., J. Aguilar Á. y J. R. Altamirano C. (2010). Los Prestadores de Servicios Profesionales como Complementadores en la Red de Valor. Universidad Autónoma de ChapingoCIESTAAM. México.

Santoyo, C., V. H., P. Ramírez y M. Suvedi. (2002). Manual para la evaluación de programas de desarrollo rural. 2 a. edición. Mundi-Prensa. 